\title{
Non-localized deformation in $\mathrm{Cu}-\mathrm{Zr}$ multi-layer amorphous films under tension
}

C. Zhong ${ }^{1}$, H. Zhang ${ }^{1,2}$, Q. P. Cao ${ }^{1}$, X. D. Wang ${ }^{1}$, D. X. Zhang ${ }^{3}$, J.W. Hu ${ }^{4}$, P. K. Liaw ${ }^{5}$ and J. Z. Jiang $^{1 *}$

${ }^{1}$ International Center for New-Structured Materials (ICNSM), Laboratory of New-Structured Materials, State Key Laboratory of Silicon Materials, and School of Materials Science and Engineering, Zhejiang University, Hangzhou, 310027, People's Republic of China

${ }^{2}$ Department of Chemical and Materials Engineering, University of Alberta, Edmonton, Alberta, T6G 1H9, Canada

${ }^{3}$ State Key Laboratory of Modern Optical Instrumentation, Zhejiang University, Hangzhou, 310027, People's Republic of China

${ }^{4}$ Hangzhou Workers Amateur University, Hangzhou, 310027, P. R. China.

${ }^{5}$ Department of Materials Science and Engineering, The University of Tennessee, Knoxville, TN 37996, USA

*) E-mail: jiangjz@,zju.edu.cn 


\begin{abstract}
In metallic glasses (MGs), plastic deformation at room temperature is dominated by highly localized shear bands. Here we report the non-localized deformation under tension in $\mathrm{Cu}-\mathrm{Zr}$ multi-layer MGs with a pure amorphous structure using large-scale atomistic simulations. It is demonstrated that amorphous samples with high layer numbers, composed of $\mathrm{Cu}_{64} \mathrm{Zr}_{36}$ and $\mathrm{Cu}_{40} \mathrm{Zr}_{60}$, or $\mathrm{Cu}_{64} \mathrm{Zr}_{36}$ and $\mathrm{Cu}_{50} \mathrm{Zr}_{50}$, present obviously non-localized deformation behavior. We reveal that the deformation behavior of the multi-layer-structured MG films is related but not determined by the deformation behavior of the composed individual layers. The criterion for the deformation mode change for MGs with a pure amorphous structure, in generally, was suggested, i.e., the competition between the elastic-energy density stored and the energy density needed for forming one mature shear band in MGs. Our results provide a promising strategy for designing tensile ductile MGs with a pure amorphous structure at room temperature.
\end{abstract}

Key words: Metallic glasses, Molecular dynamics simulations, Mechanical behavior, Non-localized deformation 


\section{Introduction}

Metallic glasses (MGs), exhibiting high strengths and large elastic limits, and unique forming capabilities in the supercooled liquid region, have been extensively investigated during the last four decades [1-6]. However, at low temperature well below the glass-transition temperature, plastic deformation of MGs is highly localized within a narrow region, so-called shear bands, resulting in the catastrophic failure and limiting the structural reliability of MGs and their applications [7-9]. Enormous efforts have been devoted in recent years to improve the plasticity of MGs. For example, MG/crystal composites have been designed by virtue of the interaction between the glass matrix and crystalline particles to inhibit the propagation of shear bands and also to promote the formation of multiple shear bands. Remarkable tensile ductility of these composites was successfully achieved [10-15]. However, improving plasticity in MGs with pure amorphous structures at low temperatures, e.g., room temperature, is still strongly demanded from both fundamental and technical points of view. Under compression, some pure amorphous-structured MGs, exhibiting large plasticity, had been developed [16-19], in which the excess open volume (or a heterogeneous structure) in samples plays a key role to create multiple shear bands and impede shear-band propagation. However, for tension under slow deformation, no evidence of room-temperature plasticity for pure amorphous-structured MGs has been reported yet. Though the mechanism of deformation behavior for MGs is still under debate [16,20,21], the heterogeneous structure concept is widely accepted to be useful to improve the plasticity of MGs. As an example, pre-plastic deformation by compression and cold rolling of MGs are introduced to create heterogeneous structures by the formation of shear bands in MGs, resulting in the improvement of the tensile plasticity of MGs [22,23]. The cold-rolled MGs even exhibited an obvious plastic elongation during tension [24,25], owing to the competitive movement of multiple shear bands in different orientations in the heterogeneously-structured MGs. Very recently, suppressing shear localization under tension in a metallic glass embedded with amorphous-structured shear bands using large-scale molecular dynamics (MD) simulations was reported [26]. They pointed a possible direction to achieve tensile non-localized deformation for the pure amorphous-structured MGs. However, the content of pre-existing shear bands requested to have the non-localized deformation is found to be as large as about $40 \%$ in volume. For the fabrication of such MGs with a high density of shear bands, it is still a problem with present available techniques. Thus, new ideas to have non-localized deformation in pure amorphous-structured MGs, which could be the experimentally achievable, are still desirable. In the present work, we propose multi-layer MG films, which are highly structural controllable and relatively easily produced in experiments, e.g., by 
the sputtering method [27-30], and report large-scale atomistic simulation results of the tensile behavior of a series of multi-layer MG films generated with different compositions in the $\mathrm{Cu}-\mathrm{Zr}$ system. It is found that the tensile deformation mode switching from highly-localized shear banding to non-localized plastic deformation is possible by varying the content and/or arrangement of components. The criterion for the deformation-mode change of MGs with pure amorphous structure, in general, was suggested. Our work shade new light on designing plastic metallic glasses with pure amorphous structure.

\section{Methods}

Using LAMMPS (Large-scale Atomic/Molecular Massively Parallel Simulator) [31], molecular dynamics simulations of $\mathrm{Cu}-\mathrm{Zr}$ MGs were conducted by the embedded atom method potential [32]. Three compositions of $\mathrm{Cu}_{64} \mathrm{Zr}_{36}$ (hereafter marked as A), $\mathrm{Cu}_{50} \mathrm{Zr}_{50}$ (hereafter marked as B), and $\mathrm{Cu}_{40} \mathrm{Zr}_{60}$ (hereafter marked as C) are selected to construct multi-layer amorphous films. Firstly, three pure A, B and C amorphous alloys with the same length scale of $28.2 \mathrm{~nm}(\mathrm{X}) \times 56.4 \mathrm{~nm}(\mathrm{Y}) \times 5.6 \mathrm{~nm}$ (Z) and 500,000 atoms are generated. The detail generation procedure of amorphous samples by the "melt-quench-duplicate" method were reported in our previous work [26,33]: a cubic cell, $5.6 \times 5.6$ $\times 5.6 \mathrm{~nm}^{3}$, with different compositions melted at $2000 \mathrm{~K}$ and zero pressure (within the uncertainty of around $10 \mathrm{MPa}$ ) for $2 \mathrm{~ns}$ to ensure homogeneity, during which periodic boundary conditions (PBCs) were applied to all three dimensions. The cell was, then, quenched from $2000 \mathrm{~K}$ to $50 \mathrm{~K}$ over $19.5 \mathrm{~ns}$ at a cooling rate of $10^{11} \mathrm{~K} / \mathrm{s}$. The small cubic cells were duplicated to form the final dimension of samples, $28.2 \mathrm{~nm}(\mathrm{X}) \times 56.4 \mathrm{~nm}(\mathrm{Y}) \times 5.6 \mathrm{~nm}(\mathrm{Z})$, then the large sample after duplication relaxed at $50 \mathrm{~K}$ for $1 \mathrm{~ns}$. Secondly, quasi-three-dimensional multi-layer films with the same size of $28.2 \mathrm{~nm}(\mathrm{X})$ $\times 56.4 \mathrm{~nm}(\mathrm{Y}) \times 5.6 \mathrm{~nm}(\mathrm{Z})$ were generated by various arrangements of pure $\mathrm{A}, \mathrm{B}$ and $\mathrm{C}$ components with different thicknesses, as shown in Fig. 1(c). To release the high excess energy associated with artificial interfaces, which were formed by putting thin layers together during sample preparation, one of the "overlapping atoms" (with a criterion of the inter-atomic distance for two atoms shorter than $0.2 \mathrm{~nm}$, which is about the shortest interatomic distance in $\mathrm{Cu}-\mathrm{Zr}$ system) at the interfaces, i.e., in total, for each interface about 1,000 atoms $(1,000 / 500,000=0.2 \%$ in total atom fraction) were removed. All multi-layer amorphous films were relaxed for 100 ps before the subsequent mechanical loading. Finally, uniaxial tensile loading was applied to all the multi-layer amorphous films and pure MGs at a constant strain rate of $1 \times 10^{8} \mathrm{~s}^{-1}$ in the $\mathrm{Y}$ direction to $20 \%$ at $50 \mathrm{~K}$. During deformation, 
PBCs were applied to the $\mathrm{Y}$ and $\mathrm{Z}$ directions, while the free-surface condition was applied to the $\mathrm{X}$ direction to mimic the film structure. The time step was chosen as $1 \mathrm{fs}$. To visualize plastic shearing during deformation, the local shear invariant [34] Von Mises strain of each atom in the sample was

calculated using the equation: $\eta^{\text {Mises }}=\sqrt{\frac{1}{2} \operatorname{Tr}\left(\eta-\eta_{m} \mathrm{I}\right)^{2}}$, where $\eta$ and $\eta_{m}$ are the local Lagrangian strain and local hydrostatic strain for that atom, respectively. To calculate the $\eta_{i}^{\text {Mises }}$ of each atom, we use the atoms in the present configuration to compare with the state before deformation. Atoms, with a Von Mises value larger than 0.2 , are illustrated to visualize the deformation process. For clarification, all studied multi-layer films here are set to be symmetric along the thickness direction.

\section{Results}

The structures and mechanical properties of $\mathrm{Cu}-\mathrm{Zr}$ MGs with different compositions have been investigated [35,36]. It is found that $\mathrm{Cu}_{64} \mathrm{Zr}_{36}$ (A) has more $\mathrm{Cu}$-centered full icosahedra (FI) and exhibits highest strengths, i.e., more resistance to the initiation of flows but increased propensity to strain localization, while $\mathrm{Cu}_{40} \mathrm{Zr}_{60}(\mathrm{C})$ is on the opposite side, and $\mathrm{Cu}_{50} \mathrm{Zr}_{50}$ (B) is in the middle. Thus, $\mathrm{A}$ and $\mathrm{C}$, with the largest difference in mechanical properties, were firstly selected to generate multi-layer amorphous films, in which $\mathrm{A}$ is considered to be the hard component, and $\mathrm{C}$ is the soft component. The questions to be addressed here are whether non-localized deformation could be observed in multi-layer amorphous films constructed by $\mathrm{A}$ and $\mathrm{C}$ components and what kind of the designing strategy could be used to enhance plasticity.

\subsection{Deformation behavior of CAC-type three-layer structured MG films}

Firstly, the CAC-type three-layer structured films, in which the A component is in the middle and two pieces of the $\mathrm{C}$ component in both sides, were studied. As shown in Fig. 1(d), here CA(50)C means that the A component located in the middle has $50 \%$ in volume and both sides of the $\mathrm{C}$ component are the same to be $25 \%$. A series of CAC-type three-layer structured samples with various volume fractions of the A component, i.e., $20 \%, 26 \%, 30 \%, 33.3 \%, 40 \%, 50 \%$ and $66.7 \%$, are generated, relaxed, and then deformed, as described in the Method section. Fig. 1(a) shows the stress-strain (SS) curves for the $\mathrm{CA}(\mathrm{X}) \mathrm{C}$ three-layer amorphous films, together with the pure $\mathrm{A}$ and $\mathrm{C}$ films for comparison. The macroscopic yield stress, i.e., the maximum stress in the SS curves, 
increases as the volume percentage of the A component gradually increases from $20 \%$ to $66.7 \%$ in Fig. 1(b), which is close to a linear relationship with volume fraction. However, it is obvious that the interface(s) formed in multi-layer MG films will reduce the strength of films, as compared to the pure amorphous components without the interface, as shown by the two arrows in Fig. 1(b). One A/C interface might reduce nearly $0.1 \mathrm{GPa}$ in strength. The detailed analysis of interface to strength will be further clarified in the discussion part. As seen in Fig. 1(a), after the yield stress, in all CAC-type multi-layer films, the stress drop rapidly, and no one reaches a stress plateau, which is similar to the pure $\mathrm{A}$ and $\mathrm{C}$ components. This trend means that shear banding occurs in all these samples during deformation. Figs. 1(c) and 1(d) show the schematic diagrams of the two-selected CA(33.3)C and $\mathrm{CA}(50) \mathrm{C}$ films and their deformation patterns at a strain of $14 \%$, respectively, in which only atoms with strains greater than 0.2 are illustrated. It clearly reveals that one dominant shear band penetrates in both samples, representing an obviously-localized shear-banding feature. These results demonstrate that although the CAC-type sandwich structure successfully modifies the strength of multi-layer MG films, it still does not change the deformation mode, suggesting that the studied three-layer structure may not be high enough in terms of the degree of heterogeneity in samples.

\subsection{Deformation behavior of CAC-type structured MG films with high layer numbers}

Secondly, we studied the films with higher layer numbers, i.e., five, seven, and nine layers, in which the total volume fractions of the $\mathrm{A}$ and $\mathrm{C}$ components are fixed to a constant value for comparison. Here we select $50 \%$ in volume as a prototype system. Meanwhile, the C component is located at the outermost, and two components arranged alternately. The schematic diagrams of $\mathrm{CA}(25) \mathrm{CA}(25) \mathrm{C}$ with 5 layers [hereafter marked as $\mathrm{CAC}(5)]$ and $\mathrm{CA}(16.7) \mathrm{CA}(16.7) \mathrm{CA}(16.7) \mathrm{C}$ with 7 layers [hereafter marked as CAC(7)] are shown in Figs. 2(c) and 2(d), respectively. As an example, in the seven-layer structured film, the C component occupied four layers and each layer has $12.5 \%$ in volume, and the A component occupied three layers and each layer has $16.7 \%$ in volume. For comparison, the two-layer structured AC film, in which each component has the content of 50\% in volume, is also built and investigated. Fig. 2(a) shows the SS curves of a series of multi-layer structured films together with the pure A and C films. A striking observation here is that for 7- and 9-layer films, after the maximum stress, stress drops very slowly, forming a plateau, while other films exhibit a typical localized shear-banding feature without a plateau. Since the stress plateau in the SS curves is one character of the non-localized deformation [26], it seems that the CAC-type multi-layer 
films with seven and nine layers exhibit relative non-localized deformation. Two deformation patterns at a strain of $14 \%$ are shown in Fig. 2(c) for the five-layer sample and Fig. 2(d) for the seven-layer sample to represent the deformation mode. The deformation patterns of both CAC(7) and $\mathrm{CAC}(9)$ at strain of $50 \%$ were also examined in Fig. S1, confirming that non-localized deformation indeed happen in both samples. These results suggest that one dominant shear band clearly penetrates the samples when layer numbers are two to five, while none dominant shear band, i.e., many deformed spots, occur and result in relative non-localized deformation when the layer number is seven or nine. It is obvious that different layers correspond to different interface numbers, e.g., nine layers correspond to eight interfaces. The relation of yield stresses and interface numbers in different CAC-type multi-layer films are presented in Fig. 2(b). The macroscopic yield stress decreases as the layer number increases from zero to nine. Here "zero" represents film without interface, which value is equal to half of the sum strength value of the pure $\mathrm{A}$ and $\mathrm{C}$ components. The higher number of the interface in multi-layer films, the lower the strength, and also the higher the degree of heterogeneity, which is helpful to the deformation mode switch from the localization to relatively-non-localized deformation.

\subsection{Deformation behavior of ACA-type structured MG films}

As the shear bands usually initialized from the surface region, we further investigate the outermost component effect on the deformation behavior of multi-layer $\mathrm{Cu}-\mathrm{Zr}$ MG films. Fig. 3(a) shows three SS curves for AC(X)A-type three-layer structured films, $\mathrm{X}=33.3 \%, 50 \%$, and $66.7 \%$, where the A component located at the outermost, together with $\mathrm{CA}(\mathrm{X}) \mathrm{C}$-type three-layer films for comparison. It is found that the SS curves of three-layer MG films $\mathrm{CA}(33.3) \mathrm{C}$ and $\mathrm{AC}(66.7) \mathrm{A}$, and $\mathrm{CA}(66.7) \mathrm{C}$ and $\mathrm{AC}(33.3) \mathrm{A}$ are almost overlapped. For the $\mathrm{CA}(50) \mathrm{C}$ and $\mathrm{AC}(50) \mathrm{A}$ two films, their SS curves show a slight difference for strains higher than $10 \%$. But, one dominant shear band was found to penetrate in all three studied ACA-type three-layer MG films, which is consistent with the results observed for the CAC-type three-layer MG films in Fig. 1. The deformation pattern of $\mathrm{AC}(50) \mathrm{A}$ at a strain of $14 \%$ is shown in Fig. 3(c). From the above results for the three-layer MG films, it seems that if the compositions are similar, they exhibit similar strength and the same deformation mode. We further investigated five, seven, and nine-layer ACA-type MG films with a composition of $50 \% \mathrm{~A}$ and $50 \% \mathrm{C}$ in volume. The SS curves of the ACA-type multi-layer MG films are presented in Fig. 3(b), together with the CAC-type multi-layer MG films for comparison. The SS 
curves for both the ACA-type and CAC-type multi-layer MG films are almost overlapped. The deformation patterns of $\operatorname{ACA}(7)$ and $\operatorname{ACA}(9)$ at strains of $14 \%$ and $50 \%$ exhibit the same non-localized deformation mode. But for the $\mathrm{ACA}(5)$ and $\mathrm{CAC}(5)$ multi-layer MG films, differences in the SS curves above the maximum stress were detected, which is consistent with the results obtained for $\mathrm{CA}(50) \mathrm{C}$ and $\mathrm{AC}(50) \mathrm{A}$ three-layer MG films. Deformation patterns from $8 \%$ to $14 \%$ for $\mathrm{ACA}(3), \mathrm{CAC}(3), \operatorname{ACA}(5)$, and $\mathrm{CAC}(5)$ are examined and found that if the harder phase, $\mathrm{A}$, locates outermost for both 3-layer and 5-layer samples, the flow stresses at a strain of 14\% are much higher than those for $\mathrm{CAC}(3)$ and $\mathrm{CAC}(5)$ films. But, localized deformation indeed happens for all four samples. Fig. 3(d) illustrates the deformation pattern of $\operatorname{ACA}(5)$ at a strain of $14 \%$, clearly demonstrating the localized shear banding behavior. Based on the above-obtained results, it can be concluded that the surface effect is not a key factor to achieve the non-localized deformation.

\section{Discussion}

\subsection{Composition effect}

The above-obtained results imply that the layer number is a key parameter to switch the deformation mode while the layer arrangement of the hard or soft component plays a minor role, when the overall sample thickness is a constant. To further check this conclusion, we replace $\mathrm{Cu}_{40} \mathrm{Zr}_{60}$ ( $\mathrm{C}$ component) by $\mathrm{Cu}_{50} \mathrm{Zr}_{50}$ (B component) and also perform tensile deformation for ABA-type multi-layer MG films. Figs. 4(a) and 4(b) show the SS curves and strength of $\mathrm{AB}(\mathrm{X}) \mathrm{A}$-type 3-layer MG films with $33.3 \%, 50 \%$, and $66.7 \%$ of the $\mathrm{B}$ component in volume, together with the pure A and B components for comparison. Similar to the results obtained for the AC(X)A-type 3-layer MG films in Fig. 3, the macroscopic yield stresses of the AB(X)A-type 3-layer MG films also decreases with the volume fraction of the B component and exhibit a localized shear-banding deformation mode (not shown). Fig. 4(c) presents the SS curves for 5-, 7- and 9-layer ABA-type MG films with both A and B components fixed as $50 \%$ in volume, together with the pure $\mathrm{A}$ and $\mathrm{B}$ components for comparison. In all these samples, the A component is located at the outermost, and two components arranged alternately. The dependence of the yield stress with the layer number is shown in Fig. 4(d), exhibiting a linear relationship, i.e., the higher the number, the lower the yield stress. Similar to seven-layer and nine-layer of ACA-type and CAC-type MG films, the non-localized deformation behavior was also observed in seven- and nine-layer ABA-type MG films at strains up to $50 \%$. The inset of Fig. $4 \mathrm{c}$ for a strain of $14 \%$ suggests that the strategy, i.e., a 
high number of the multi-layer-type structured MG films, can be used to design amorphous samples, exhibiting non-localized deformation during tension.

\subsection{Interface characterization}

By comparing multi-layer with single-layer films, besides the different structure of each component, interfaces in multi-layer films are the most important factor. Thus, we need to carefully characterize the structures and properties of the interface. When constructing the multi-layer film by putting two components together, it is inevitable that some atoms are located closely, then one of such atoms with a distance less than $0.2 \mathrm{~nm}$ was removed. In total, about 1,000 atoms are removed in each interface. The detailed structures of the interface, including the potential energy (PE), composition, density and polyhedral structure, are still needed to be further studied. Small regions near the interface, with a size of $4 \mathrm{~nm}(\mathrm{X}) \times 8 \mathrm{~nm}(\mathrm{Y}) \times 5.6 \mathrm{~nm}(\mathrm{Z})$ in the AC(33.3)A-type 3-layer film, are selected to be analyzed. The region, as a typical example, is marked by blue rectangle in the inset of Fig. 5(b), and could be artificially divided into 80 small sub-domains along $X$ axis. As a result, their properties, averaged by all atoms within each sub-domain, could be plotted as a function of their atomic distance to the interface. Firstly, Fig. 5(a) shows the potential energy (PE) at both before and after relaxation states. As $\mathrm{Cu}$ atoms have much higher $\mathrm{PE}$ than $\mathrm{Zr}$ atoms, it is reasonable to consider them separately. Before relaxation, it is obvious that the average PEs of atoms near the interface with a width of about $0.8 \mathrm{~nm}$ are much higher than those in the matrix. However, after relaxation, the PE values near the interface largely decreased to almost that in the matrix, confirming that the relaxation process is very effective to stable the interface. The insets in Fig. 5(a) highlight the average PE value of atoms after relaxation. It seems that the interface is reasonably constructed. Fig. 5(b) shows the composition, i.e., the percent of $\mathrm{Cu}$ atoms in each sub-domain, as a function of the distance along $\mathrm{X}$ axis after relaxation. At the left side, the average content of $\mathrm{Cu}$ is nearly $64 \%$ in the A-component region and at right side it is nearly $40 \%$ in the $\mathrm{C}$-component region. Meanwhile, at the interface within a narrow width of about $0.8 \mathrm{~nm}$, the average $\mathrm{Cu}$ content gradually decreases from $64 \%$ to $40 \%$, which is in line with our expectations. Furthermore, the average density as a function of the distance along $\mathrm{X}$ axis after relaxation is shown in Fig. 5(c). Although the density presents a relatively large fluctuation, in the A-component region, it is close to $7.584 \mathrm{~g} / \mathrm{cm}^{3}$, close to 6.964 $\mathrm{g} / \mathrm{cm}^{3}$ in the C-component region and at the interface region, the density gradually changes. It is commonly believed that MGs are composed of different types of polyhedra, which have a wide 
distribution in terms of the types and fractions of polyhedra $[35,36]$. In the $\mathrm{Cu}-\mathrm{Zr}$ binary MG system, the $\mathrm{Cu}$-centered full icosahedra (FI) with Voronoi index $[0,0,12,0]$ is believed as a key polyhedral $[33,37]$, which varied with the composition. The fraction of the $\mathrm{Cu}$-centered FI as a function of the distance along $X$ axis after relaxation is shown in Fig. 5(d), revealing a gradual decrease along the interface region from $25 \%$ on the left side in the A-component region to $8 \%$ on the right side in the C-component region. From the above results, including PE, composition, density, and polyhedral structure analyses, it can be concluded that the interface constructed here is reasonable, and our results on the deformation behavior of multi-layer MG films are expected to be reliable.

\subsection{Interface effect}

To further study the interface effect, we carried out two more simulations. (1) After 100 ps relaxation, we relax the sample further for another $100 \mathrm{ps}$ at $50 \mathrm{~K}$. The SS curve of the 200 ps-relaxed sample in Fig. 6(a) exhibits the similar strength and the same localized deformation behavior as the former only 100 ps-relaxed sample. This trend confirms that the 100 ps relaxing process already stables the interface. (2) To respond to the suspicion that many atoms at interface were removed during construction of multi-layer films, one 3-layer film sample composed of $\mathrm{AC}(33.3) \mathrm{A}$ and 12 cylindrical holes at the interface is constructed, as shown in Fig. 6(b), the diameter of holes is randomly selected to be $0.4 \mathrm{~nm}$. In this sample, nearly 2,000 atoms (about 1,000 atoms from cylindrical holes) per interface, which is about $2,000 / 500,000=0.4 \%$ in the total atom fraction, are removed. After 100 ps relaxation, this sample performed similar tensile deformation, and the SS curve is also shown in Fig. 6(a). It is clear that even doubling the number of atoms being removed, the strength and deformation mode remain almost the same. These results clearly indicate that the removal of atoms at each interface during the construction of multi-layer films has minor effects to the switch of the deformation mode from highly-localized shear banding to the relatively non-localized deformation behavior. From above analysis, it can be further confirmed that the $100 \mathrm{ps}$ relaxation is effective to construct reasonable interfaces. Thus, the decrease in the strength of the CAC or ABA three-layer films with two interfaces, as shown in Figs. 2(b) and 4(b), could be reasonably attributed to the finite thickness interfaces, rather than moving several atoms when constructing the interfaces.

The next issue that we considered is how the interface affects the deformation of the multi-layer structured MG films, especially the initialization and evolution of a shear band. Fig. 7 shows 
deformation patterns for various multi-layer structured $\mathrm{MG}$ films together with pure $\mathrm{A}$ and $\mathrm{C}$ components at various strains, in which only atoms with a Von Mises value larger than 0.2 , defined as "S-atoms", are illustrated. It is clear that at a strain of $8 \%$, close to the maximum stress, most S-atoms are found at interfaces and surfaces, i.e., the interface regions could be considered as soft regions, as compared to the matrix. The higher the layer number, the more S-atoms in the multi-layer structured MG films. With increasing strain, many small-sized shear bands, marked by red circles in the seven-layer sample, are broken at the interfaces, which prevent the formation of one dominant shear band with increasing strains at $13 \%$ (even up to $20 \%$, not shown), exhibiting the non-localized deformation behavior. But, for samples with layer numbers below 7, one narrow region, marked by dash lines, in which many S-atoms are located, are detected at a strain of $13 \%$, exhibiting the localized deformation behavior. It is found that the more interfaces, the more $\mathrm{S}$ atoms are formed, which promote the formation of many locally deformed zones (or shear transformation zones) in the whole sample, consequently inhibiting the formation of one dominant shear band during deformation up to high stain level.

\subsection{Size effect}

All multi-layer MG films studied here are composed of small sub-domains of the pure A component, B component or $\mathrm{C}$ component. The higher the layer number, the smaller the width of each component along the $\mathrm{X}$ axis. We will next discuss the size effect on the deformation-mode change in our multi-layer MG films. In fact, numerous experimental and computational studies have demonstrated that deformation mode change could be attained in MGs by reducing the sample size [38-49]. Very recently, we performed a series of uniaxial tension simulations on the pure components of $\mathrm{A}, \mathrm{B}$ and $\mathrm{C}$ with various widths [47], i.e., only the width along the $\mathrm{X}$ axis changed from $28.2 \mathrm{~nm}$ to nearly $3 \mathrm{~nm}$. It was revealed that with decreasing the film width, the deformation-mode change from the localized to the non-localized deformation indeed occurs in all three $\mathrm{A}, \mathrm{B}$, and $\mathrm{C}$ components, which could be a general phenomenon for any MGs. [46,47] The critical thickness is composition-dependent, which is detected to be $3.33 \pm 0.20 \mathrm{~nm}, 4.95 \pm 0.25 \mathrm{~nm}$ and $6.77 \pm 0.25 \mathrm{~nm}$ for the component $\mathrm{A}, \mathrm{B}$, and $\mathrm{C}$, respectively. The deformation mode of a series of multi-layer $\mathrm{MG}$ films composed by A and C, or A and B, combined with the deformation mode of each sub-domain, were summarized in Table I. It is found that when each individual MG component in multi-layer MG films has a size larger than their corresponding critical sizes, the multi-layer MG films deformed 
locally. On the other hand, when each individual MG component in multi-layer MG films has a size smaller than their corresponding critical sizes, the multi-layer MG films deformed non-locally. For multi-layer MG films, in which only one component has a size smaller than its corresponding critical size, deformation behavior becomes complicated. For example, in the CA(66.7)C three-layer film, the sub-domain of the $\mathrm{C}$ component $(4.7 \mathrm{~nm})$ is below its critical size $(6.77 \mathrm{~nm})$, but the multi-layer MG film still shows a localized deformation. The CAC(7), ACA(7) and CAC(9) multi-layer structured films, although the sub-domain of the A component has sizes larger than its critical size $(3.33 \mathrm{~nm})$, still show the non-localized deformation behavior. Similar results were also observed in the multi-layer MG films composed by A and B components. From the results listed in Table I, we may find that if all sub-domains in the multi-layer films are localized or non-localized, the multi-layer structured MG films will show similar deformation behavior. However, if one sub-domain is localized while the other is non-localized, either a localized or non-localized deformation feature is possible in the multi-layer structured MG films. Thus, it seems that the deformation modes of the multi-layer structured MG films are related but not determined by the deformation modes of the composed individual layers. Thus, only the size effect is inadequate to explain the non-localized deformation observed in the seven-layer or nine-layer MG films mentioned above.

\subsection{Energy analysis}

In our previous work [47], the width-dependent deformation-mode transition in MGs was determined by the competition of the stored elastic energy density, $U_{p}$, and the energy density needed for forming one mature shear band, $\mathrm{U}_{\mathrm{SB}}$. It is found that the localized deformation through the shear band occurs only if the total energy that is stored in the sample at the onset of plastic deformation, $U_{p}$, is equal to or larger than $\mathrm{U}_{\mathrm{SB}}$. If $\mathrm{U}_{\mathrm{p}}<\mathrm{U}_{\mathrm{SB}}$, the stored energy in the sample is insufficient to cause the localization, the MG sample deforms in a non-localized mode [47]. Thus, we calculate $U_{S B}$ and $U_{p}$ for studied the multi-layer MG films in Fig. 8(a). As illustrated in Ref. 47, the S-atoms start to appear at the maximum stress of the SS curve labeled "a", i.e., the notable plastic deformation starts at this strain. When the sample is strained to the point marked by the arrow, one mature shear band is formed. Thus, it is reasonable to assume that the strain energy that is spent during deformation of the sample from the point, "a", to "arrow", is the energy required to localize deformation. Two solid straight lines are drawn parallel to the initial linear elastic part of the SS curve at points, "a", and 
"arrow". Before the formation of a mature shear band, the energy dissipated (the area under the SS curve up to the arrow) can be partitioned into three parts, labeled as $A_{1}, A_{2}$, and $A_{3}$. The energy density in the region, $\mathrm{A}_{1}$, contributes to the local inelastic deformation and dissipated throughout the whole sample. The energy encompassed by the region, $A_{3}$, corresponds to the recoverable part by unloading from the arrow, i.e., it is the elastic strain energy that remains in the sample even after a shear band was formed. Therefore, the energy that is spent during the formation of a mature shear band is the region, $A_{2}$, defined as $U_{S B}$. The values of $U_{p}$, representing the energy stored in samples before the start of plastic deformation, were calculated by the area of the SS curves from zero to the point, "a", in Fig. 8(b) together with $\mathrm{U}_{\mathrm{SB}}$ values for $\mathrm{AC}, \mathrm{CAC}(3)$, and $\mathrm{CAC}(5) \mathrm{MG}$ films. It is found that films below 7 layers, i.e., below 6 interfaces, $\mathrm{U}_{\mathrm{p}}>\mathrm{U}_{\mathrm{SB}}$, indicating a localized deformation, as observed in Fig. 2. For films with 6 or 8 interfaces, i.e., 7 or 9 layers, $U_{p}$ is indeed smaller than $U_{S B}$, indicating a non-localized deformation, as observed in Fig. 2. Therefore, the criterion of the relative energy values of $U_{p}$ and $U_{S B}$ is able to explain the deformation mode change observed in our studied multi-layer MG films, which is in agreement with previous results [47] for single-layer MGs.

\subsection{Sample width effect}

Because the above-obtained multi-layer samples have a fix width of $28.2 \mathrm{~nm}$, thus, it is still desirable to examine the width effect. Considering that the deformation mode of multi-layers strongly correlate to their specific width in each sub-domain, we use the following strategy: without changing each layer width, to test whether increasing or decreasing the layer numbers, i.e., increasing or decreasing the sample width, could affect its deformation mode. As shown in Fig. 9, the specific deformation mode of the width-altered multi-layer films could be ensured from both the stress-strain curves and deformation maps at a strain of 14\%. In Fig. 9(a), when the layer numbers of the uniform-deformed ACA(9) (marked as "a9") reduce to 5 or 7 (marked as "a9-5" or "a9-7") with the total sample widths of $15.5 \mathrm{~nm}$ and $21.9 \mathrm{~nm}$, respectively, it will still be uniformly deformed in both cases. On the contrary, when the layer numbers of the localized-deformed ACA(5) (marked as "a5") extend to 7 (marked as "a5-7") with the total sample width of $39.95 \mathrm{~nm}$, the localized deformation still occurs, as shown in Fig. 9(b). These results in Fig. 9 clearly suggest that the enhancement or reduction of the layer number will not alter their deformation-mode. In all $28.2 \mathrm{~nm}$-thick multi-layers, we have confirmed that the localized deformation through shear bands occurs only if the total energy that is stored in the sample at the onset of plastic deformation, $U_{p}$, is equal to or larger than $U_{\mathrm{SB}}$. $I n$ 
Fig. 9, it is obtained that the value of $U_{p}$ remains almost unchanged, due to the nearly overlapping of whose SS curves before the maximum stress, as shown in Figs. 9(a) and 9(b). Meanwhile, the value of $U_{S B}$ is also similar when changing the layer numbers without altering their specific width in each sub-domain, because their volume fractions of both $\mathrm{A}$ and $\mathrm{C}$ components in the entire multi-layer samples are nearly the same. Therefore, the criterion of the relative energy values of $U_{p}$ and $U_{S B}$ is also able to explain the deformation-mode change observed in these width-altered multi-layer MG films. We believe that this criterion is generally validated for the deformation-mode change for MGs with pure amorphous structures.

\subsection{Comparison to the real world}

In the section 4.4 , it is found that the deformation modes of the multi-layer structured MG films are related to the deformation modes of the composed individual layers. We note that the values of critical thickness to be typically less than $10 \mathrm{~nm}$ in simulations [46,47] are much smaller than the experimentally obtained ones to be typically in the range of 50 to $300 \mathrm{~nm}[48,49]$. This discrepancy could be mainly due to the following possible reasons. (a) The effective strain rate, $10^{8} \mathrm{~s}^{-1}$, which is often used for MD simulations, is much larger than the rates of $10^{-2}-10^{-4} \mathrm{~s}^{-1}$ used in many experiments. (b) In experiments MG samples always contain external defects/flaws while the samples tested through MD simulations do not contain any external defects. (c) The samples prepared in MD simulations are quenched by a cooling rate of about $10^{11} \mathrm{~K} / \mathrm{s}$, while most bulk metallic glasses prepared by casting are quenched by a cooling rate of about $10^{2} \mathrm{~K} / \mathrm{s}$. This means that the samples in experiment relax much more than these in simulation, consequently resulting in lower content of "free volume" and more brittle in experimental samples. Hence, based on the above-mentioned facts, a direct comparison of results obtained from MD simulations with the real world is still difficult and more studies, e.g., strain rate and cooling rate effects on deformation mode change in MGs by MD simulations, are strongly suggested. However, the finding obtained here by MD simulations points out one direction for experiments to design ductile MGs with multi-layer structure.

\section{Conclusions}

In summary, we have performed MD simulations to systematically investigate the uniaxial tensile deformation behavior of multi-layer $\mathrm{MG}$ films composed of $\mathrm{Cu}_{64} \mathrm{Zr}_{36}$ and $\mathrm{Cu}_{40} \mathrm{Zr}_{60}$, or 
$\mathrm{Cu}_{64} \mathrm{Zr}_{36}$ and $\mathrm{Cu}_{50} \mathrm{Zr}_{50}$. It is revealed that amorphous multi-layer samples with high layer numbers (e.g., 7 and 9 layers) could have non-localized deformation behavior up to tensile strain of 50\%. The deformation behavior of the multi-layer-structured MG films is related but not determined by the deformation behavior of the composed individual layers. The criterion for the deformation mode change for MGs with a pure amorphous structure, in generally, was suggested, i.e., the competition between the elastic-energy density stored and the energy density needed for forming one mature shear band in MGs. These results shed the new light on designing tensile ductile MGs with a pure amorphous structure at room temperature.

\section{Acknowledgements}

Financial supports from the National Key Basic Research Program of China (2012CB825700), National Natural Science Foundation of China (grants 51371157 and U14321056), Natural Science Foundation of Zhejiang Province (grants Z1110196 and Y4110192), and the Fundamental Research Funds for the Central Universities are gratefully acknowledged. PKL would like to acknowledge the Department of Energy (DOE), Office of Fossil Energy, National Energy Technology Laboratory (DE-FE-0008855, DE-FE-0024054, and DE-FE-0011194), with Mr V. Cedro, Mr. R. Dunst, and J. Mullen as program managers. PKL appreciates the support of the U.S. Army Research Office project (W911NF-13-1-0438) with the program manager, Dr. D.M. Stepp. PKL thanks the support from the National Science Foundation (CMMI-1100080) with the program director, Dr. C. Cooper. The computer resources at National Supercomputer Center in Tianjin and the calculations performed on TianHe-1 (A) are also gratefully acknowledged. 
References:

[1] A. Inoue, Stabilization of metallic supercooled liquid and bulk amorphous alloys, Acta Mater. 48 (2000) 279-306.

[2] W.H. Wang, C. Dong, C.H. Shek, Bulk metallic glasses, Mater. Sci. Eng. R. 44 (2004) 45-89.

[3] A.L. Greer, E. Ma, Bulk metallic glasses: at the cutting edge of metals research, MRS Bulletin. 32 (2007) 611-615.

[4] Y.Q. Cheng, E. Ma, Atomic-level structure and structure-property relationship in metallic glasses, Prog. Mater. Sci. 56, (2011) 379-473.

[5] A. Inoue, B. L. Shen, H. Koshiba, H. Kato, A.R. Yavari, Cobalt-based bulk glassy alloy with ultrahigh strength and soft magnetic properties, Nat. Mater. 2 (2003) 661-663.

[6] Q.K. Jiang, P. Liu, Y. Ma, Q.P. Cao, X.D. Wang, D.X. Zhang, X.D. Han, Z. Zhang, and J.Z. Jiang, Super elastic strain limit in metallic glass films, Sci. Rep. 852 (2012) 1-6.

[7] M.W. Chen, Mechanical behavior of metallic glasses: Microscopic understanding of strength and ductility, Annu. Rev. Mater. Res. 38 (2008) 445-469.

[8] C.A. Schuh, T.C. Hufnagel, U. Ramamurty, Mechanical behavior of amorphous alloys, Acta Mater. 55 (2007) 4067-4109.

[9] B. Yang, M.L. Morrison, P.K. Liaw, R.A. Buchanan, G.Y. Wang, C.T. Liu, M. Denda, Dynamic evolution of nanoscale shear bands in a bulk-metallic glass, Appl. Phys. Lett. 86 (2005) 141904.

[10] C.C. Hays, C.P. Kim, W.L. Johnson, Microstructure controlled shear band pattern formation and enhanced plasticity of bulk metallic glasses containing in situ formed ductile phase dendrite dispersions, Phys. Rev. Lett. 84 (2000) 2901-2904.

[11] D.C. Hofmann, J.Y. Suh, A. Wiest, G. Duan, M.L. Lind, M.D. Demetriou, W.L. Johnson, Designing metallic glass matrix composites with high toughness and tensile ductility, Nature, 451 (2008) 1085-1090.

[12] J.W. Qiao, A.C. Sun, E.W. Huang, Y. Zhang, P.K. Liaw, C.P. Chuang, Tensile deformation micromechanisms for bulk metallic glass matrix composites: From work-hardening to softening, Acta Mater. 59 (2011) 4126-4137.

[13] J.W. Qiao, H.L. Jia, P.K. Liaw, Bulk metallic glass matrix composites, Mater. Sci. Eng. R. In press. 
[14] A. Donohue, F. Spaepen, R.G. Hoagland, A. Misra, Suppression of the shear band instability during plastic flow of nanometer-scale confined metallic glasses, Appl. Phys.Lett. 91 (2007) 241905.

[15] Y.M. Wang, J. Li, A.V. Hamza, T.W. Barbee Jr, Ductile crystalline-amorphous nanolaminates, Proc. Natl. Acad. Sci. U.S.A. 104 (2007) 11155-11160.

[16] Y.H. Liu, G. Wang, R.J. Wang, M.X. Pan, W.H. Wang, Super plastic bulk metallic glasses at room temperature, Science 315 (2007) 1385-1388.

[17] J. Schroers, W.L. Johnson, Ductile bulk metallic glass, Phys. Rev. Lett. 93 (2004) 255506.

[18] M.W. Chen, A. Inoue, W. Zhang, T. Sakurai, Extraordinary plasticity of ductile bulk metallic glasses, Phys. Rev. Lett. 96 (2006) 245502.

[19] L.Y. Chen, Z.D. Fu, G.Q. Zhang, X.P. Hao, Q.K. Jiang, X.D. Wang, Q.P. Cao, H. Franz, Y.G. Liu, H.S. Xie, S.L. Zhang, B.Y. Wang, Y.W. Zeng, J.Z. Jiang, New Class of Plastic Bulk Metallic Glass, Phys. Rev. Lett. 100 (2008) 075501.

[20] L.Y. Chen, Y.W. Zeng, Q.P. Cao, B.J. Park, Y.M. Chen, K. Hono, U. Vainio, Z.L. Zhang, U. Kaiser, X.D. Wang, J.Z. Jiang, Homogeneity of the $\mathrm{Zr}_{64.13} \mathrm{Cu}_{15.75} \mathrm{Ni}_{10.12} \mathrm{Al}_{10}$ bulk metallic glass, J. Mater. Res. 24 (2009) 3116-3120.

[21] Y.H. Liu, W.H. Wang. Comment on "Homogeneity of $\mathrm{Zr}_{64.13} \mathrm{Cu}_{15.75} \mathrm{Ni}_{10.12} \mathrm{Al}_{10}$ bulk metallic glass,” by L-Y. Chen, Y-W. Zeng, Q-P. Cao, B-J. Park, Y-M. Chen, K. Hono, U. Vainio, Z-L. Zhang, U. Kaiser, X-D. Wang, and J-Z. Jiang [J. Mater. Res. 24, 3116 (2009)], J. Mater. Res. 25 (2010) 598-601.

[22] Y. Yokoyama, K. Yamano, K. Fukaura, H. Sunada, A. Inoue. Ductility improvement of $\mathrm{Zr}_{55} \mathrm{Cu}_{30} \mathrm{Al}_{10} \mathrm{Ni}_{5}$ bulk amorphous alloy, Scripta Mater 44 (2001) 1529-1533.

[23] Y. Yokoyama, Ductility improvement of Zr-Cu-Ni-Al glassy alloy, J. Non-Cryst. Solids. 316 (2003) 104-113.

[24] Y. Yokoyama, K. Fukaura, A. Inoue. Cast structure and mechanical properties of Zr-Cu-Ni-Al bulk glassy alloys, Intermetallics 10 (2002) 1113-1124.

[25] Q.P. Cao, J.W. Liu, K.J. Yang, F. Xu, Z.Q. Yao, A. Minkow, H.J. Fecht, J. Ivanisenko, L.Y. Chen, X.D. Wang, S.X. Qu, J.Z. Jiang, Effect of pre-existing shear bands on the tensile mechanical properties of a bulk metallic glass, Acta Mater 58 (2010) 1276-1292.

[26] H.F. Zhou, C. Zhong, Q.P. Cao, S.X. Qu, X.D. Wang, W. Yang, J.Z. Jiang, Non-localized deformation in metallic alloys with amorphous structure, Acta Mater. 68 (2014) 32-41. 
[27] S.Y. Kuan, H.S. Chou, M.C. Liu, X.H. Du, J.C. Huang, Micromechanical response for the amorphous/amorphous nanolaminates, Intermetallics. 18 (2010) 2453-2457.

[28] P. Sharma, K. Yubuta, H. Kimura, A. Inoue, Brittle metallic glass deforms plastically at room temperature in glassy multilayers, Phys. Rev. B 80 (2009) 024106.

[29] J.P. Chu, J.S.C. Jang, J.C. Huang, H.S. Chou, Y. Yang, J.C. Ye, Y.C. Wang, J.W. Lee, F.X. Liu, P.K. Liaw, Y.C. Chen, C.M. Lee, C.L. Li, C. Rullyani, Thin film metallic glasses: Unique properties and potential applications, Thin Solid Films 520 (2012) 5097-5122.

[30] H.L. Jia, F.X. Liu, Z.N. An, W.D. Li, G.Y. Wang, J.P. Chu, J.S.C. Jang, Y.F. Gao, P.K. Liaw, Thin-film metallic glasses for substrate fatigue-property improvements, Thin Solid Films 561 (2014) $2-27$.

[31] S. Plimpton. Fast parallel Algorithms for short-range molecular dynamics, J. Comput. Phys. 117 (1995) 1-42.

[32] M.I. Mendelev, D.J. Sordelet, M.J. Kramer, Using atomistic computer simulations to analyze X-ray diffraction data from metallic glasses, J. Appl. Phys. 102 (2007) 043501.

[33] A.J. Cao, Y.Q. Cheng, E. Ma, Structural processes that initiate shear localization in metallic glass, Acta Mater. 57 (2009) 5146-5155.

[34] F. Shimizu, S. Ogata, J. Li, Theory of shear banding in metallic glasses and molecular dynamics calculations, Mater. Trans. 48 (2007) 2923-2927.

[35] Y.Q. Cheng, A.J. Cao, H.W. Sheng, E. Ma, Local order influences initiation of plastic flow in metallic glass: Effects of alloy composition and sample cooling history, Acta Mater. 56 (2008) 5263-5275.

[36] Y.Q. Cheng, H.W. Sheng, E. Ma, Relationship between structure, dynamics, and mechanical properties in metallic glass-forming alloys, Phys. Rev. B 78 (2008) 014207.

[37] Y. Ritter, D. Sopu, H. Gleiter, K. Albe, Structure, stability and mechanical properties of internal interfaces in $\mathrm{Cu}_{64} \mathrm{Zr}_{36}$ nanoglasses studied by MD simulations, Acta Mater. 59 (2011) 6588-6593.

[38] H. Guo, P.F. Yan, Y.B. Wang, J. Tan, Z.F. Zhang, M.L. Sui, E. Ma, Tensile ductility and necking of metallic glass, Nat. Mater. 6 (2007) 735-739.

[39] D.C. Jang, J.R. Greer, Transition from a strong-yet-brittle to a stronger-and-ductile state by size reduction of metallic glasses, Nat. Mater. 9 (2010) 215-219. 
[40] J.R. Greer, J.T.M. De Hosson, Plasticity in small-sized metallic systems: Intrinsic versus extrinsic size effect, Prog. Mater. Sci. 56 (2011) 654-724.

[41] J.H. Luo, F. F. Wu, J.Y. Huang, J.Q. Wang, S.X. Mao, Superelongation and atomic chain formation in nanosized metallic glass, Phys. Rev. Lett. 104 (2010) 215503.

[42] C.Q. Chen, Y.T. Pei, J.T.M. De Hosson, Effects of size on the mechanical response of metallic glasses investigated through in situ TEM bending and compression experiments, Acta Mater. 58, (2010) 189-200.

[43] Y. Ma, Q.P. Cao, S. X. Qu, X.D. Wang, J.Z. Jiang, Effect of structural relaxation on plastic flow in a Ni-Nb metallic glassy film, Acta Mater. 60 (2012) 3667-3676.

[44] Y. Ma, Q.P. Cao, S.X. Qu, X.D. Wang, D.X. Zhang, J.Z. Jiang, Stress-state-dependent deformation behavior in Ni-Nb metallic glassy film, Acta Mater. 60 (2012) 4136-4143.

[45] Q.P. Cao, C. Wang, K.J. Bu, S.Y. Liu, X.D. Wang, D.X. Zhang, J.Z. Jiang, The influence of glass transition temperature on the critical size for deformation mode transition in metallic glassy films, Scripta Mater. 77 (2014) 64-67.

[46] C. Zhong, H. Zhang, Q. P. Cao, X. D. Wang, D. X. Zhang, J. Z. Jiang, The size-dependent non-localized deformation in a metallic alloy, Scripta Mater. 101 (2015) 48-51.

[47] C. Zhong, H. Zhang, Q. P. Cao, X. D. Wang, D. X. Zhang, U. Ramamurty, J. Z. Jiang, On the critical thickness for non-localized to localized plastic flow transition in metallic glasses: a molecular dynamics study, Scripta Mater. 114 (2016) 93-97.

[48] C.Q. Chen, Y. T. Pei, O. Kuzmin, Z. F. Zhang, E. Ma, J.T.M. De Hosson, Intrinsic size effects in the mechanical response of taper-free nanopillars of metallic glass. Phys. Rev. B. 83 (2011) 180201.

[49] O.V. Kuzmin, Y.T. Pei, C.Q. Chen, J.T.M. De Hosson, Intrinsic and extrinsic size effects in the deformation of metallic glass Nanopillars. Acta Mater. 60 (2012) 889-898. 


\section{Table Caption:}

Table I: The deformation mode in a series of multi-layer MG films composed by A and C, or A and $\mathrm{B}$, together with the deformation mode in each individual sub-domain. The critical thicknesses for the deformation mode change for the individual A, B and C MGs are 3.33 $\pm 0.20,4.95 \pm 0.25$ and $6.77 \pm$ $0.25 \mathrm{~nm}$, respectively. 
Figure Captions

Fig. 1(a) Stress-strain curves of a series of CAC-type three-layer MG films. (b) Strength with different contents of the A component in a series of CAC-type three-layer MG films. (c) Schematic diagram of $\mathrm{CA}(33.3) \mathrm{C}$ and its deformation pattern at a strain of $14 \%$. (d) Schematic diagram of $\mathrm{CA}(50) \mathrm{C}$ and its deformation pattern at a strain of $14 \%$. Only atoms with the Von Mises strain large than 0.2 were illustrated.

Fig. 2(a) Stress-strain curves of a series of multi-layer MG films with different layers. (b) Strength with different interface numbers in a series of multi-layer MG films. (c) Schematic diagram of $\mathrm{CAC}(5)$ and its deformation pattern at a strain of 14\%. (d) Schematic diagram of CAC (7) and its deformation pattern at a strain of $14 \%$. Only atoms with the Von Mises strain large than 0.2 were illustrated.

Fig. 3(a) Stress-strain curves of a series of CAC-type and ACA-type three-layer MG films. (b) Stress-strain curves in a series of CAC-type and ACA-type multi-layer MG films. (c) Schematic diagram of $\mathrm{AC}(50) \mathrm{A}$ and its deformation pattern at a strain of 14\%. (d) Schematic diagram of ACA (5) and its deformation pattern at a strain of 14\%. Only atoms with the Von Mises strain large than 0.2 were illustrated.

Fig. 4(a) Stress-strain curves of a series of ABA-type three-layer MG films. (b) Strength with different contents of the B component in a series of ABA-type three-layer MG films. (c) Stress-strain curves in a series of ABA-type multi-layer MG films. (Inset) Deformation patterns of ABA(7) and $\operatorname{ABA}(9)$ at a strain of $14 \%$. Only atoms with the Von Mises strain large than 0.2 were illustrated. (d) Strength with different interface numbers in a series of ABA-type multi-layer MG films.

Fig. 5(a) Average potential energy (PE) of the atoms with different distances to the interface before and after relaxation. (Inset) Magnification of PE with different distances to the interface after relaxation. (b) Composition. (Inset) One typical region in AC(33.3)A marked by the blue rectangle for the interface structural analysis. (c) Density and (d) Fraction of the Cu-centered full icosahedra (FI) as a function of the distance to the interface after relaxation. 
Fig. 6(a) Stress-strain curves of the $\mathrm{AB}(33.3) \mathrm{A}$ relaxed for $100 \mathrm{ps}$ and 200 ps respectively, and also $\mathrm{AB}(33.3) \mathrm{A}$ with cylindrical holes at the interface. (b) Schematic diagram of $\mathrm{AB}(33.3) \mathrm{A}$ with 12 cylindrical holes at the interface.

Fig. 7 Deformation patterns of $\mathrm{ACA}(7), \mathrm{ACA}(5), \mathrm{ACA}(3)$, pure $\mathrm{C}$ and $\mathrm{A}$ components from $8 \%$ to $13 \%$. Only atoms with the Von Mises strain large than 0.2 were illustrated. The red circles point the local deformation prevented by the interfaces, and the dash lines mark the shear band in samples.

Fig. 8(a) Stress-strain curves of AC, CAC(3), and CAC(5) multi-layers and schematic illustration of the procedure utilized for estimating $\mathrm{U}_{\mathrm{SB}}$. (b) Variation of $\mathrm{U}_{\mathrm{p}}$ as a function of interface numbers. The dashed line represents the value of $\mathrm{U}_{\mathrm{SB}}$ in layer-structured $50 \% \mathrm{~A} / 50 \% \mathrm{C} \mathrm{MG}$ films with one dominant shear band.

Fig. 9(a) Stress-strain curves of "a9", "a9-7" and "a9-5" MG films. (Inset) Deformation patterns of "a9", "a9-7" and "a9-5" MG films at a strain of 14\%. "a9" is the ACA(9) 28.2 nm-width 9-layer sample $[\mathrm{A}(10) \mathrm{C}(12.5) \mathrm{A}(10) \mathrm{C}(12.5) \mathrm{A}(10) \mathrm{C}(12.5) \mathrm{A}(10) \mathrm{C}(12.5) \mathrm{A}(10)]$, "a9-7" is the $21.855 \mathrm{~nm}$-width 7-layer sample with actual volume composition of [A(12.9)C(16.1)A(12.9)C(16.1)A(12.9) $\mathrm{C}(16.1) \mathrm{A}(12.9)]$, and "a9-5" is the $15.51 \mathrm{~nm}$-width 5-layer sample with actual volume composition of $[\mathrm{A}(18.2) \mathrm{C}(22.7) \mathrm{A}(18.2) \mathrm{C}(22.7) \mathrm{A}(18.2)]$. (b) Stress-strain curves "a5" and "a5-7" MG films. (Inset) Deformation patterns of "a5" and "a5-7" at a strain of 14\%. "a5" is the ACA(5) 28.2 nm-width 5-layer sample $[\mathrm{A}(16.7) \mathrm{C}(25) \mathrm{A}(16.7) \mathrm{C}(25) \mathrm{A}(16.7)]$, "a5-7" is the $39.95 \mathrm{~nm}$-width 7-layer sample with actual volume composition of $[\mathrm{A}(11.8) \mathrm{C}(17.6) \mathrm{A}(11.8) \mathrm{C}(17.6) \mathrm{A}(11.8) \mathrm{C}(17.6) \mathrm{A}(11.8)]$. Only atoms with Von Mises strain large than 0.2 were illustrated. 

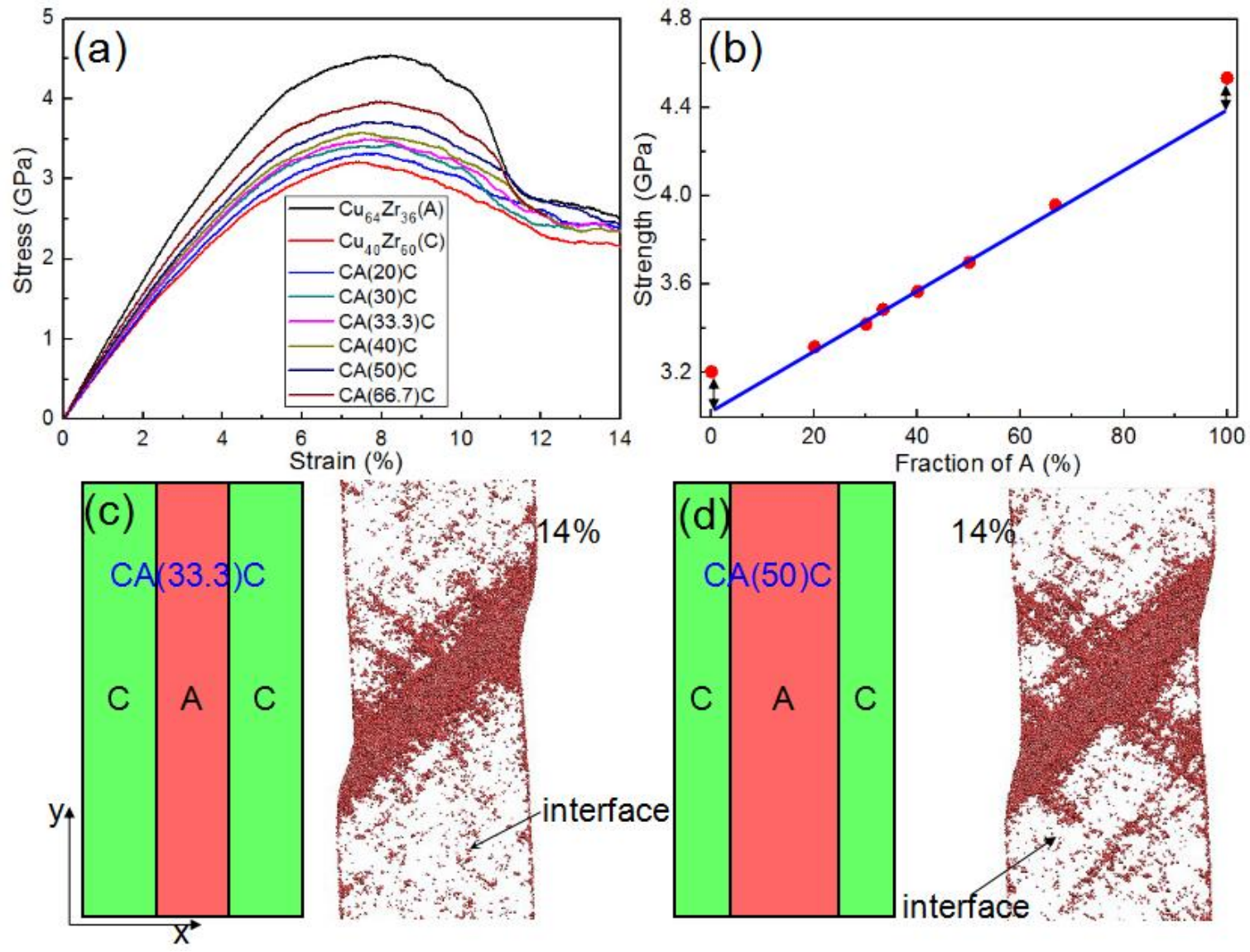

Fig .1 

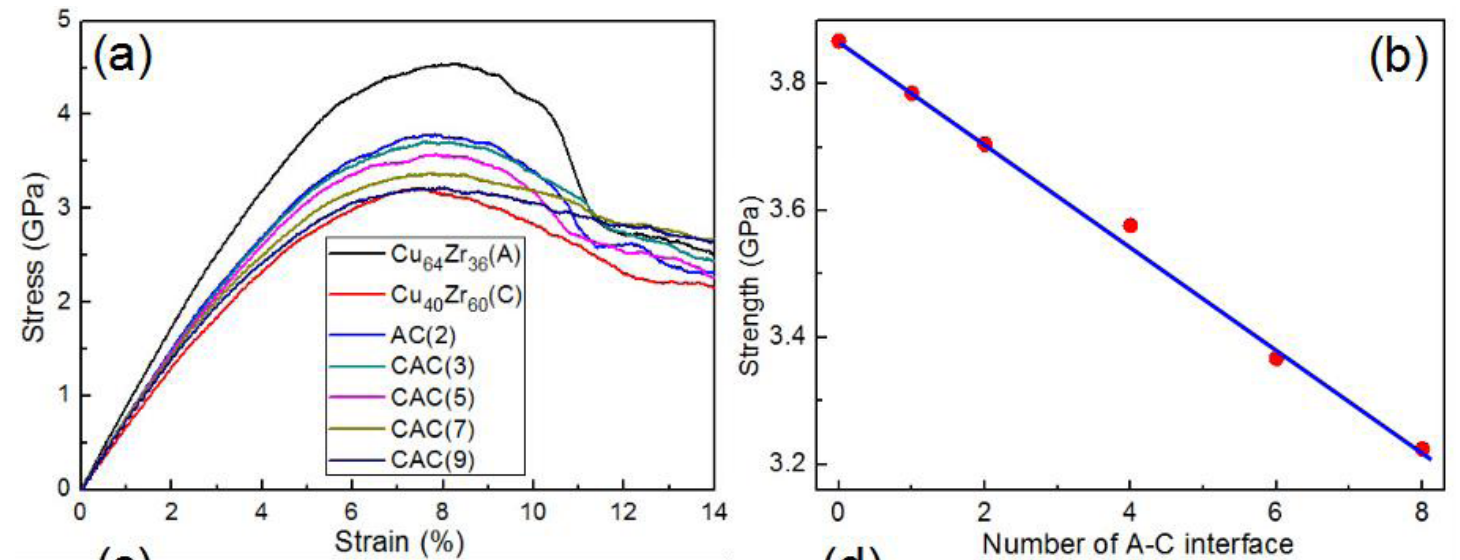

(c)
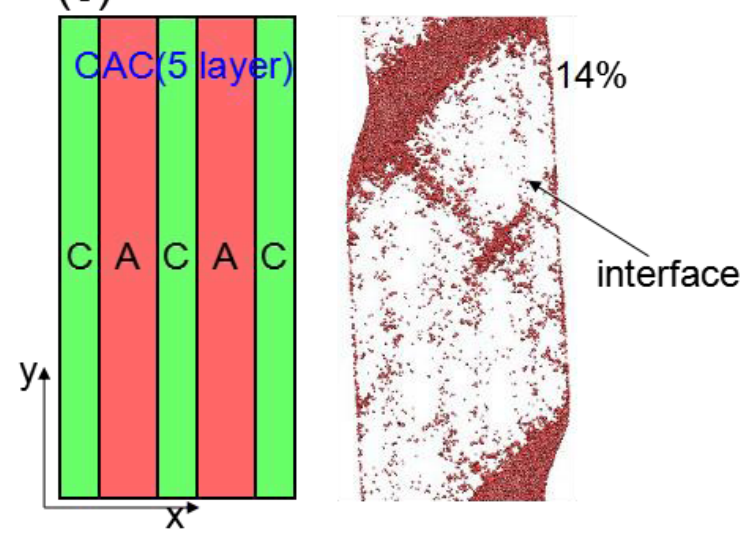

(d)

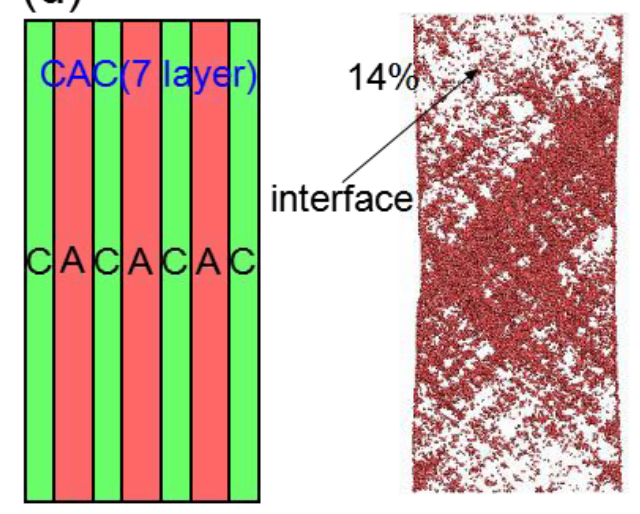

Fig .2 

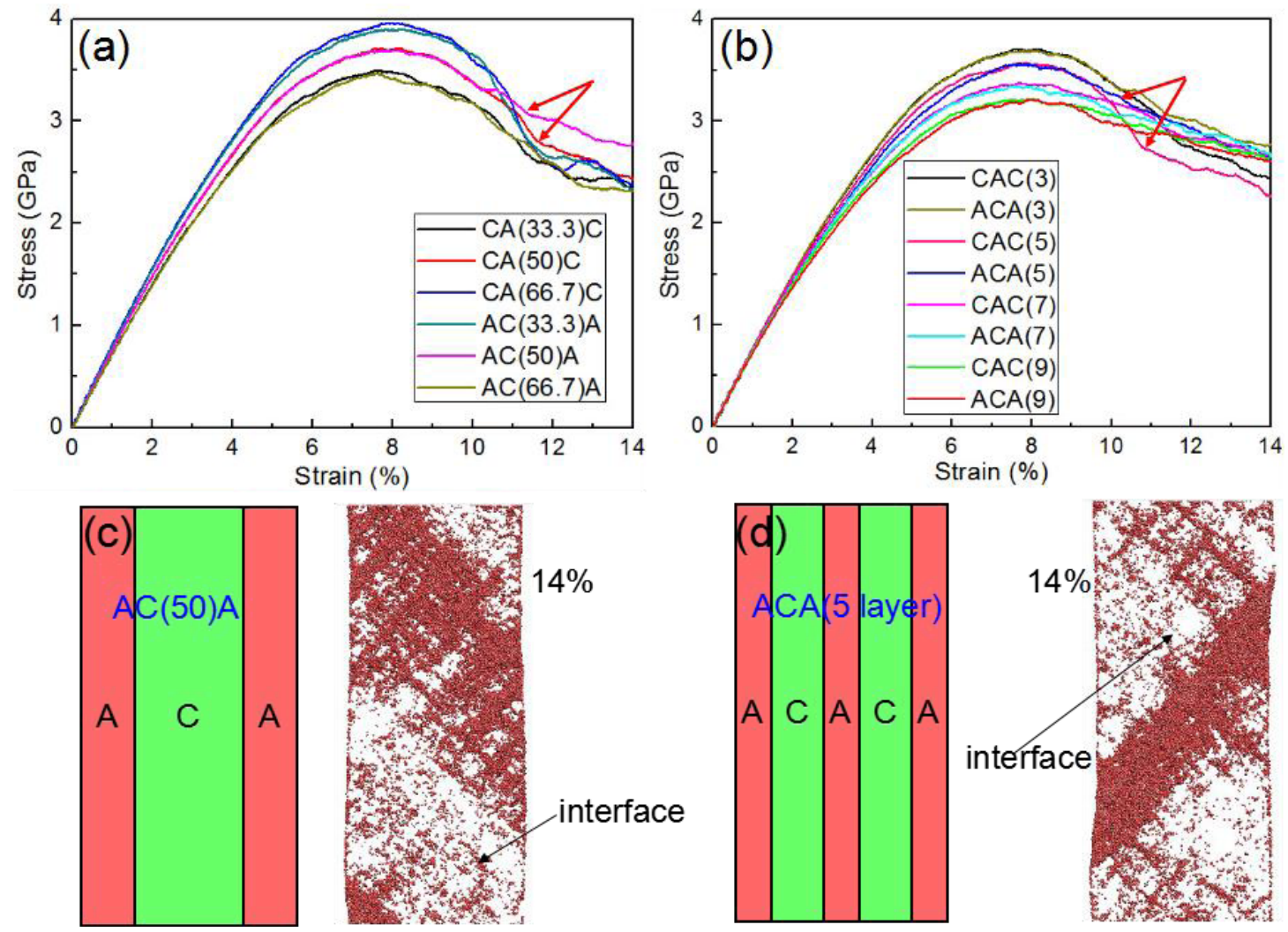

Fig .3 

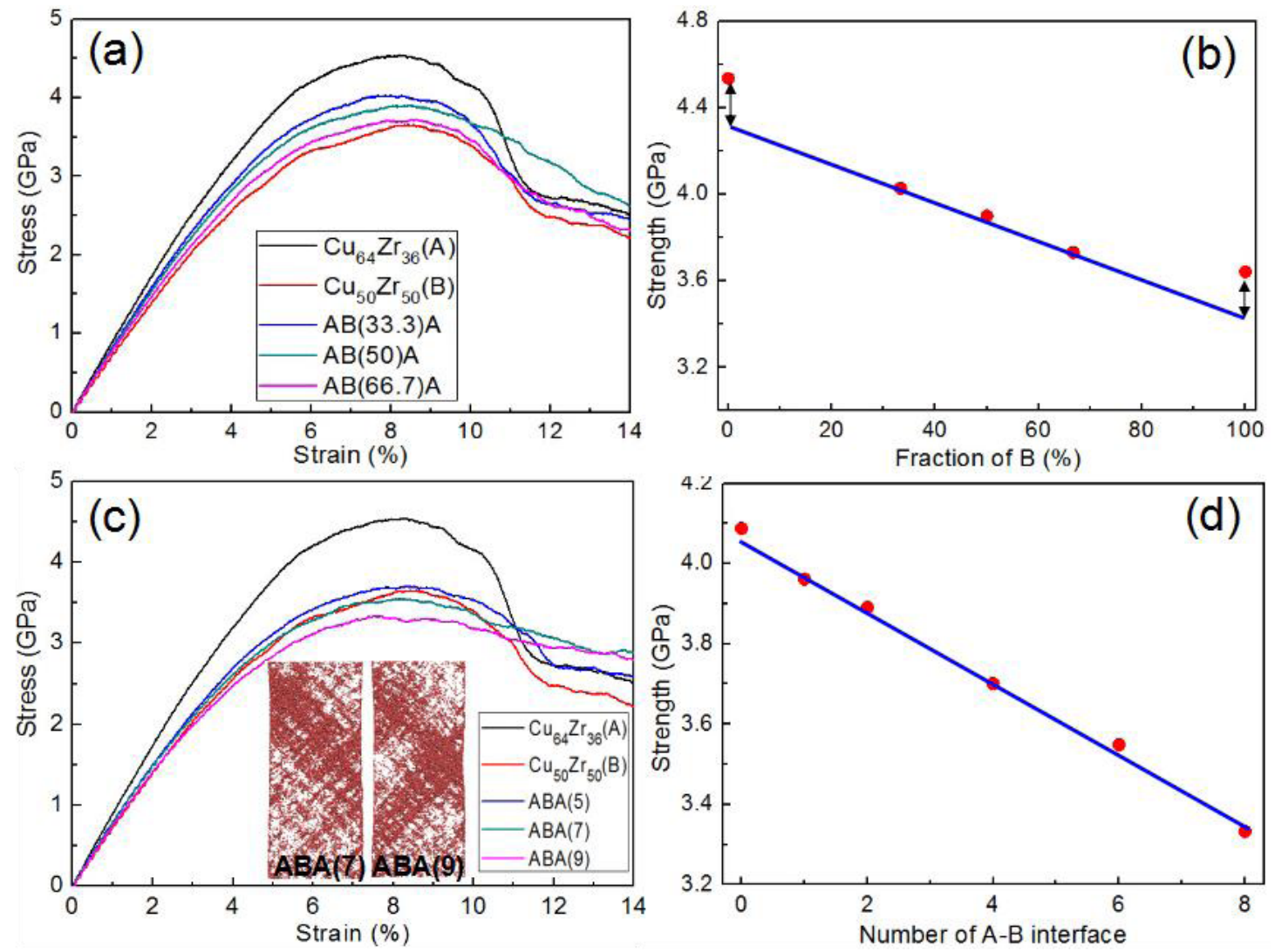

Fig .4 

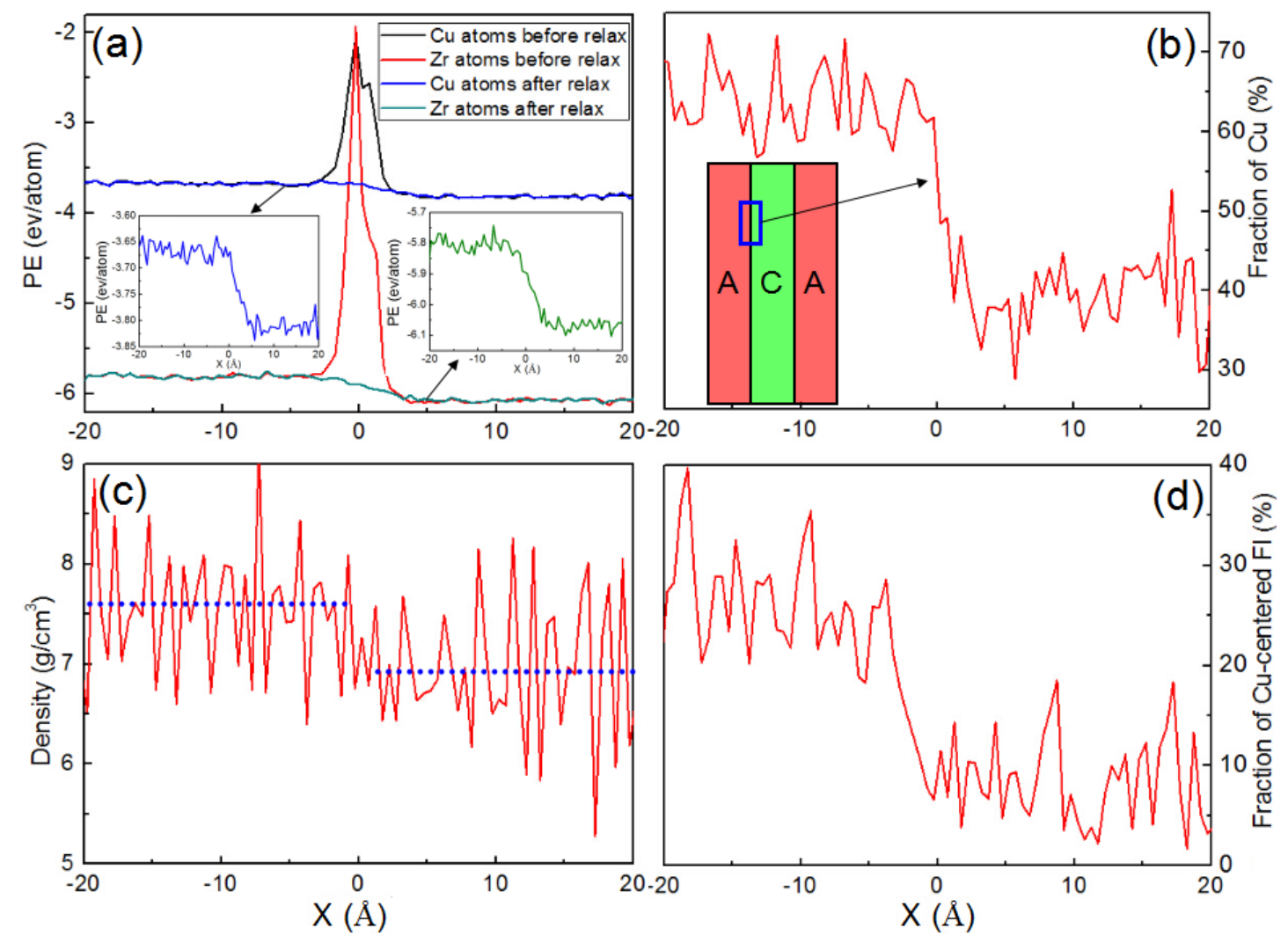

Fig .5 

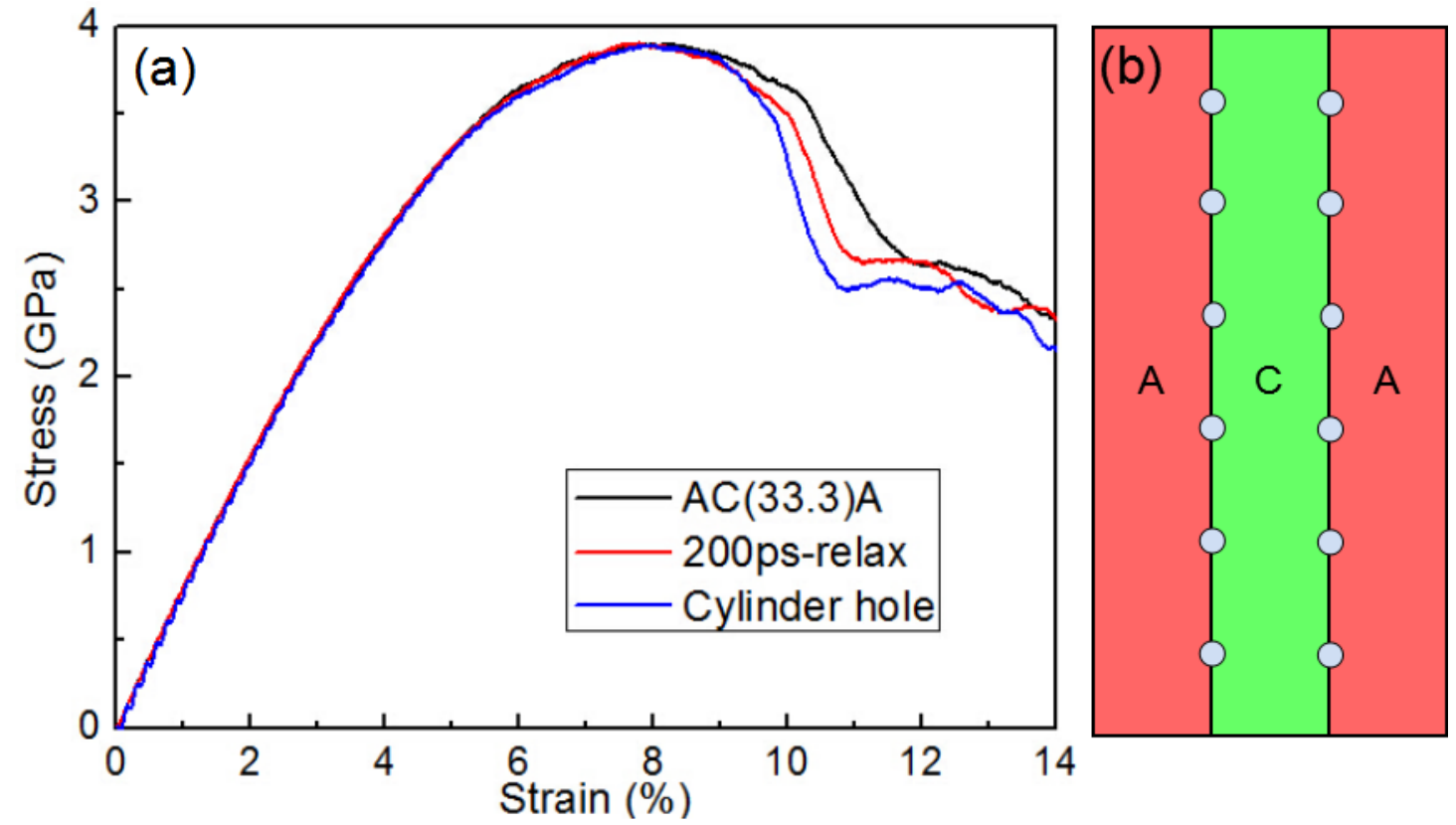

Fig .6 


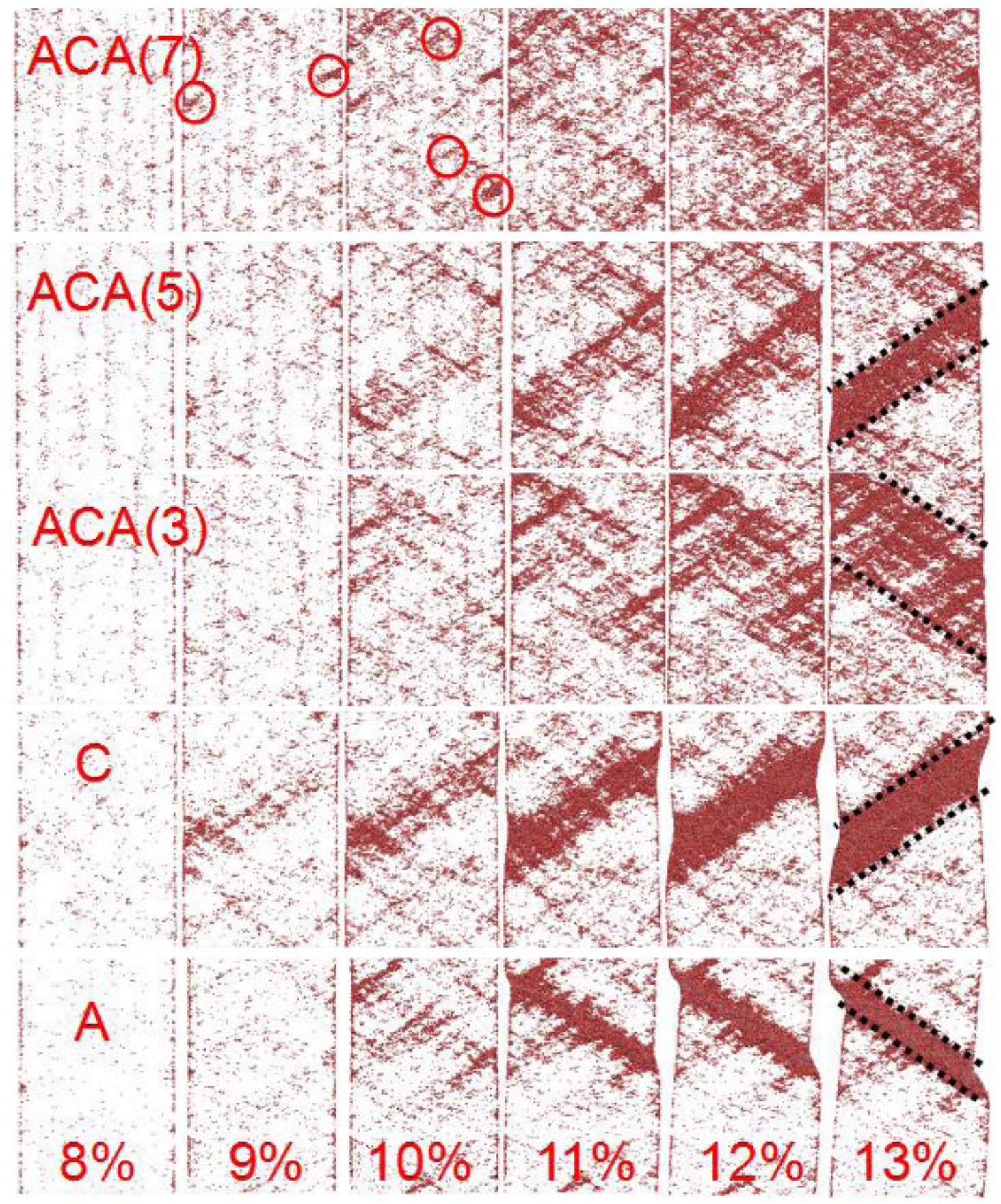

Fig .7 

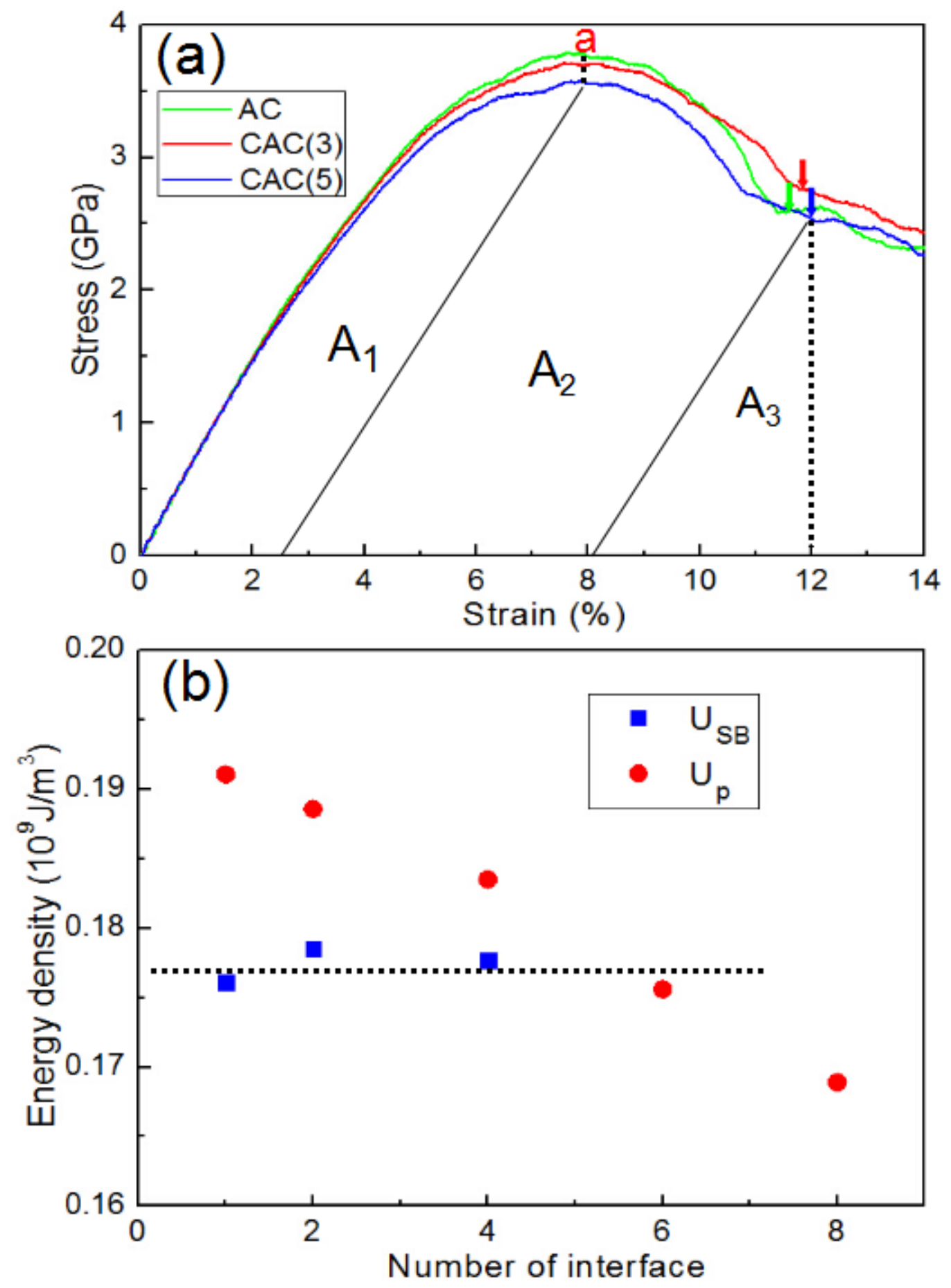

Fig .8 

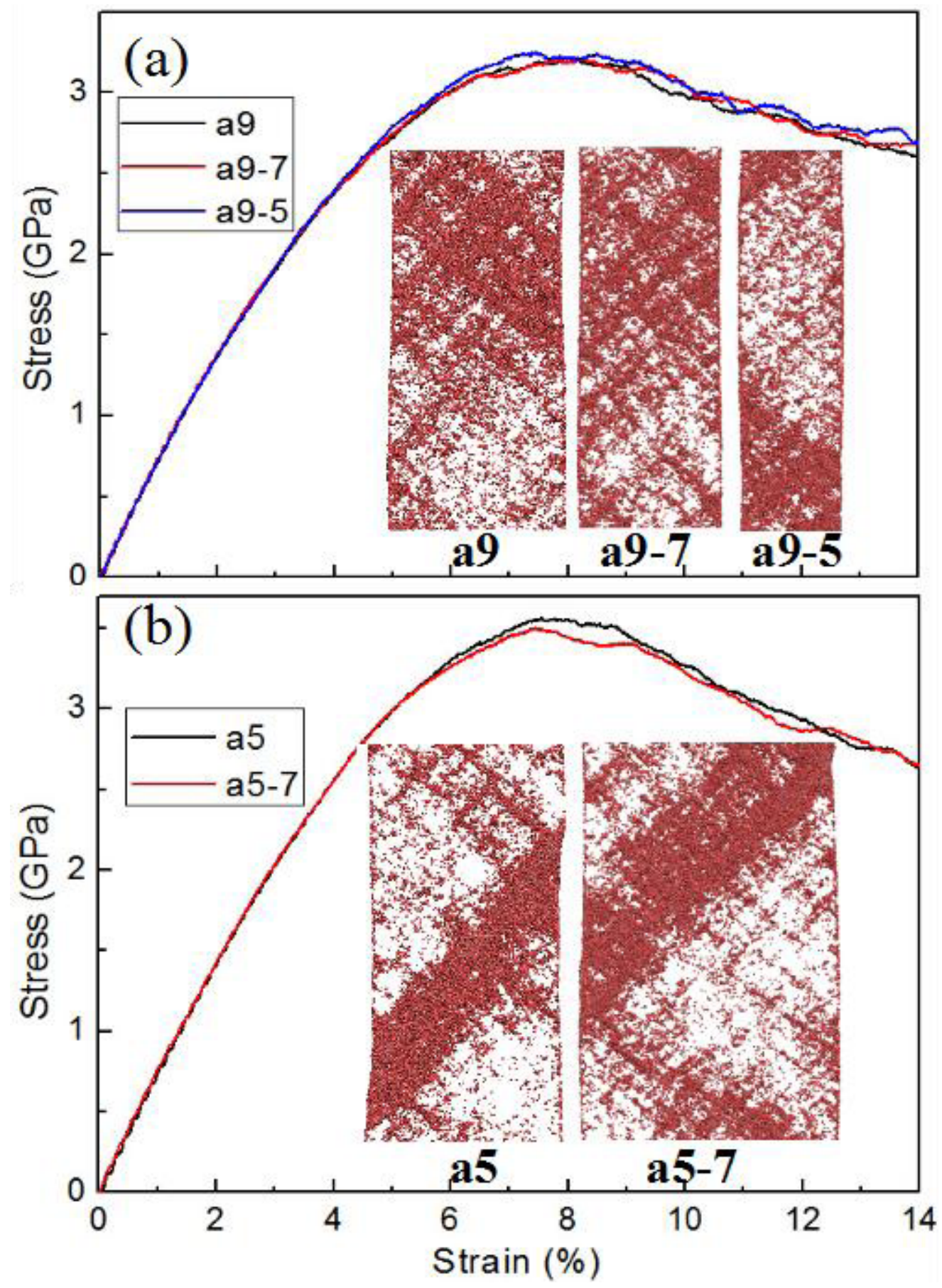

Fig .9 


\begin{tabular}{|c|c|c|c|c|c|}
\hline & $\begin{array}{l}\text { Width of } \\
\text { domain A } \\
\text { (nm) }\end{array}$ & $\begin{array}{l}\text { Non- } \\
\text { localization in } \\
\text { domain A }\end{array}$ & $\begin{array}{l}\text { Width of } \\
\text { domain C } \\
\text { or B (nm) }\end{array}$ & $\begin{array}{l}\text { Non- } \\
\text { localization in } \\
\text { domain C or B }\end{array}$ & $\begin{array}{l}\text { Non- } \\
\text { localization in } \\
\text { multi-film }\end{array}$ \\
\hline $\mathrm{CA}(33.3) \mathrm{C}$ & 9.4 & No & 9.4 & No & No \\
\hline $\mathrm{CA}(50) \mathrm{C}$ & 14.1 & No & 7.05 & No & No \\
\hline $\mathrm{CA}(66.7) \mathrm{C}$ & 18.8 & No & 4.7 & Yes & No \\
\hline $\mathrm{AC}(33.3) \mathrm{A}$ & 9.4 & No & 9.4 & No & No \\
\hline$A C(50) A$ & 7.05 & No & 14.1 & No & No \\
\hline $\mathrm{AC}(66.7) \mathrm{A}$ & 4.7 & No & 18.8 & No & No \\
\hline $\mathrm{CAC}(5)$ & 7.05 & No & 4.7 & Yes & No \\
\hline $\mathrm{ACA}(5)$ & 4.7 & No & 7.05 & No & No \\
\hline $\mathrm{CAC}(7)$ & 4.7 & No & 3.525 & Yes & Yes \\
\hline $\operatorname{ACA}(7)$ & 3.525 & No & 4.7 & Yes & Yes \\
\hline $\mathrm{CAC}(9)$ & 3.525 & No & 2.82 & Yes & Yes \\
\hline $\operatorname{ACA}(9)$ & 2.82 & Yes & 3.525 & Yes & Yes \\
\hline$A B(33.3) A$ & 9.4 & No & 9.4 & No & No \\
\hline$A B(50) A$ & 7.05 & No & 14.1 & No & No \\
\hline $\mathrm{AB}(66.7) \mathrm{A}$ & 4.7 & No & 18.4 & No & No \\
\hline $\mathrm{ABA}(5)$ & 4.7 & No & 7.05 & No & No \\
\hline $\mathrm{ABA}(7)$ & 3.525 & No & 4.7 & Yes & Yes \\
\hline $\mathrm{ABA}(9)$ & 2.82 & Yes & 3.525 & Yes & Yes \\
\hline
\end{tabular}

Table I 